\title{
ORIGINAL
}

\section{CONSUMO DE MEDICAMENTOS EN LOS ANCIANOS: RESULTADOS DE UN ESTUDIO POBLACIONAL (*)}

\author{
Emiliana Valderrama Gama (1), Fernando Rodríguez Artalejo (2), Antonia Palacios Díaz (3), \\ Pilar Gabarre Orús (3) y Jesús Pérez del Molino Martín (4).
}

(1) Departamento de Epidemiología y Bioestadística. Escuela Nacional de Sanidad, Instituto de Salud Carlos III.

(2) Departamento de Medicina Preventiva y Salud Pública. Facultad de Farmacia. Universidad del País Vasco.

(3) Ayuntamiento de Villanueva de los Castillejos. Huelva.

(4) Sección de Geriatría. Hospital General y Docente de Guadalajara.

(*) Estudio parcialmente financiado por el Ayuntamiento de Villanueva de los Castillejos (Huelva)

\section{RESUMEN}

Fundamento: Evaluar la magnitud, naturaleza y cronicidad del consumo de medicamentos en ancianos no institucionalizados, así como la complejidad de su régimen terapéutico

Método: Estudio transversal a través de encuesta a domicilio de todas las personas de 65 o más años. Municipio de Villanueva de los Castillejos (Huelva). El entrevistador pedía a la persona que mostrara todos los medicamentos que usaba sin olvidar ninguno, anotaba el nombre comercial, dosis y frecuencia con que el medicamento era consumido, y el tiempo aproximado en meses o años en que lo venía utilizando. Se introducen métodos de medida para evaluar la complejidad del régimen terapéutico.

Resultados: Se obtuvo información de 362 sujetos $(83,8 \%$ de la población de hecho). El 83,1\% de ellos utlizaba uno o más medicamentos a diario, con una media (desviación estándar) de 2,6 (2.0). Los más usados fueron los antihipertensivos $(41,8 \%)$, los vasodilatadores periféricos dirigidos a la circulación cerebral $(21,3 \%)$, las benzodiacepinas $(17,4 \%)$ y los antinflamatorios no esteroideos $(14.1 \%$ ). El 91.3 y $46,1 \%$ de los sujetos con consumo diario tomaba uno o más fármacos desde hacía al menos 1 y 5 años respectivamente. La medicación era consumida como promedio entre una y dos veces al día. Aproximadamente un $20 \%$ de la población estudiada tomaba medicamentos sólo cuando aparecían ciertos síntomas, y un 7,3\% de los fármacos estaban prescritos de forma regular, pero no diaria.

Conclusiones: La cantidad y cronicidad del consumo de medicamentos es elevada. aunque comparable con el de otros estudios españoles con similar metodología El régimen de prescripción parece sencillo para las personas mayores.

Palabras clave: Ancianos. Encuesta. Enfermedades crónicas. Farmacoepidemiología. Medio rural. Prescripción de medicamentos. Utilización de medicamentos.

Correspondencia:

Emiliana Valderrama Gama

Departamento de Epidemiología y Bioestadística.

Escuela Nacional de Sanidad. Instituto de Salud Carlos III

C/Sinesio Delgado 8.

28029 Madrid.

Tel:939-45648

Correo electrónico: EMIVAL@santandersupernet.com

\section{ABSTRACT}

\section{The Use of Medications among the Elderly: Results of a Population-based Study}

Background: To evaluate the magnitude, nature and chronicity of the use of medications on the part of non-institutionalized elderly individuals as well as the complexity of their treatment routine.

Method: Cross-section study through door-to-doo: survey of all individuals age 65 or above. Municipality of Villanueva de los Castillejos (Huelva). The surveyor asked the person to show him/her all of the medications he/she was using without overlooking any, recorded the brand name, dosage and frequency with which the medication in question was taken, and the approximate length of time, in months or in years, over which said medication had been taken. Measurement methods were included for the purpose of assessing the complexity of the treatment routine.

Results: Information was gathered from 362 subjects ( $83.8 \%$ of the actual population). Of all those surveyed, $83.1 \%$ was taking one or more medications daily, for an average (standard deviation) of $2.6(2.0)$. Those used most were antihypertensive drugs $(41.8 \%)$, peripheral vasodilators for circulation to the brain $(21.3 \%)$, benzodiazepine derivatives $(17.4 \%)$ and nonsteroid antiinflammatory drugs $(14.1 \%) .91 .3 \%$ and $46.1 \%$ of the subjects who were taking medication daily, had been taking one or more drugs for at least 1 and 5 years, respectively. The medication in question was taken on the average of one to two times a day. Approximately $20 \%$ of the population surveyed was taking medication solely when certain symptoms arose, and $7.3 \%$ of the drugs were prescribed regularly, but not daily.

Conclusions: The quantity and chronicity of the consumption of medications is high, although comparable to that of other Spanish studies conducted employing similar means and methods. The routine prescribed seems simple for seniors.

Key Words: Elderly. Survey. Chronic diseases. Pharmacoepidemiology. Rural environment. Prescribing medications. Taking medications. 


\section{INTRODUCCIÓN}

Los ancianos son el grupo de población que más medicamentos consume y el más susceptible a reacciones adversas por medicamentos $^{1-3}$. Existen evidencias de que esta mayor susceptibilidad no es debida al envejecimiento y a los cambios acompañantes en la farmacodinamia y farmacocinética, sino a las enfermedades y la polifarmacia que a menudo presentan los ancianos ${ }^{4}$. La polifarmacia, justificada en muchos casos, es a menudo desproporcionada para sus problemas crónicos, a pesar de que puede ocasionar deterioro importante de la salud del anciano $^{5,6}$. Además de relacionarse con un aumento exponencial de la incidencia de efectos adversos ${ }^{3}$, predispone al incumplimiento terapéutico y dificulta hacer nuevos diagnósticos y el inicio de otros tratamientos. Entre las causas potenciales de polifarmacia figura el prescribir regímenes terapéuticos complicados ${ }^{7}$. Sin embargo, por lo que conocemos, no existen formas estandarizadas de evaluación de la complejidad (o sencillez) del régimen terapéutico.

Basándonos en la importancia de todo lo anterior, el propósito de este trabajo es analizar la cantidad, naturaleza, cronicidad y complejidad del consumo de medicamentos de los ancianos residentes en un municipio rural de Andalucía (Villanueva de los Castillejos, Huelva). Para medir la complejidad del régimen, empleamos dos formas de evaluación: la proporción de personas que consumen medicamentos periódica pero no diariamente, y la relación númcro de dosis/ número de medicamentos.

\section{SUJETOS Y MÉTODOS}

\section{Diseño y sujetos de estudio}

El estudio forma parte de otro más amplio sobre la situación sociosanitaria de los ancianos de la localidad ${ }^{8}$. Es un estudio transversal mediante encuesta de salud por entrevista a domicilio. Se consideró población objeto de estudio a todas las personas de 65 o más años del núcleo poblacional del Municipio de Villanueva de los Castillejos (Huelva). Se pretendía estudiar a toda la población, por lo que no hubo necesidad de muestreo. Al no existir residencias de ancianos en la localidad, se trata de un estudio sobre personas mayores no institucionalizadas. Se obtuvo el último padrón municipal, correspondiente a 1991. De 522 ancianos originalmente en el padrón (proporción de envejecimiento $=19,5 \%$ ) se excluyeron 41 individuos que vivían dispersos en fincas fuera del núclco poblacional. De los 481 sujetos empadronados en el núcleo de población, 49 no residían en el municipio: 10 habían fallecido, 31 vivían en otras localidades, 4 vivían en residencias de ancianos fuera de la localidad, y 4 se encontraban hospitalizados. La población anciana de hecho quedó por tanto establecida en 432 individuos. Se tomaron medidas para minimizar la tasa de no respuesta: toda la población mayor de 65 años recibió una carta del alcalde informándoles del estudio y de la importancia de su participación. De los 432 individuos, $32(7,4 \%)$ no pudieron ser localizados aún despues de haber acudido en tres ocasiones a su domicilio.

Se contactó en su domicilio con 400 individuos, de los cuales $30(6,9 \%)$ rehusaron ser entrevistados. En el caso de las personas que no estaban en condiciones de realizar la entrevista debido a problemas de lenguaje (afasia/disartria), sordera extrema o demencia se aceptó como válida la información dada por el cuidador principal (4\% de las entrevistas).

Se obtuvieron cuestionarios de 139 hombres y 231 mujeres. La información referente a medicación se encontraba completa en 362 sujetos $(83,8 \%$ de la población de hecho).

\section{Recogida y análisis de los datos}

La recogida de la información fue realizada por 15 entrevistadores no sanitarios du- 
rante los meses de febrero y marzo de 1996 , a los que se entrenó específicamente para el estudio. De las 370 personas entrevistadas, la información sobre el consumo de medicamentos estaba completa en 360 sujetos, lo que supone el $83,3 \%$ de la población anciana de hecho. La información fue obtenida de la siguiente forma: a cada persona mayor se le solicitaba que mostrara todos los medicamentos que usaba, sin olvidar los, a veces, no considerados como tales (hipnóticos, analgésicos, inhaladores, colirios, supositorios, pomadas y cremas). El entrevistador anotaba el nombre comercial, la dosis, la periodicidad con que el medicamento era consumido, y el tiempo aproximado en meses o años en que lo venía usando. Cuando se trataba de medicamentos no consumidos a diario, se preguntó si eran usados por iniciativa del individuo (esto es, cuando aparecen síntomas hacia los que van dirigidos), o si estaban prescrito de forma no diaria (ejemplo, cada 2-3 días o una vez a la semana).

Evaluamos la complejidad del régimen terapéutico con el número de dosis/número de medicamentos, y con la proporción de medicamentos prescritos periódica pero no diariamente. El número de dosis se calcula mediante la suma del número de dosis/día de cada medicamento que toma a diario. Si un sujeto toma la medicación $\mathrm{A}$ dos veces al día (por ejemplo 1 comprimido cada 12 horas), la medicación B una vez al día (por ejemplo 2 comprimidos una vez al día con el desayuno), y la medicación $C$ tres veces al día ( 1 cápsula con desayuno, comida y cena), el número de dosis es: $2+1+3=6$ y el número de dosis/número de medicamentos es $6 / 3=2$. La interpretación es que, como media, el sujeto sigue un régimen con medicamentos prescritos para tomar dos veces al día. Consideremos otro sujeto que toma las medicaciones $\mathrm{D}, \mathrm{E}$ y $\mathrm{F}$, cada una de ellas, una vez al día. El número de dosis/ número de medicamentos será: $1+1+1 / 3=$ $=3 / 3=1$. El segundo sujeto, tomando el mismo número de medicamentos que el primero, sigue un régimen más sencillo, puesto que todos los fármacos han de ser consumidos cada 24 horas. Cuánto más cercana a 1 sea la relación número de dosis/número de medicamentos, mayor número de medicamentos de una sóla toma diaria estará consumiendo el sujeto, menor número de medicamentos con varias tomas al día, y por lo tanto, menor complejidad del régimen y facilidad de cumplimiento terapéutico. Por otro lado, los medicamentos prescritos de forma periódica pero no diaria, también dificultan el cumplimiento (1) (ejemplo: pude ser más fácil para un anciano acordarse de tomar un medicamento todos los días, que los días impares de la primera quincena de cada mes, como a menudo se prescriben algunas preparaciones oftalmológicas).

Para los fármacos se hizo una clasificación por órganos y aparatos, a la que se añadió un grupo para analgésicos y otro para nutrientes.

En el análisis estadístico se usó la prueba de la chi-cuadrado (con corrección de Yates cuando fue necesario) en el contraste de proporciones, y la $t$ de Student y ANOVA de una vía para el contraste de medias. Los datos se procesaron con el programa estadístico Statview + SE Graphics TM, en un ordenador Apple LCIII.

\section{RESULTADOS}

La edad media (desviación estándar) de los sujetos fue de 74,9 (6,9) años; 73,6 para hombres y 75,0 para las mujeres $(\mathrm{p}=0,004)$.

El $83,1 \%$ de los entrevistados utilizaban uno o más medicamentos a diario, lo que supuso una media de 2,6 $(2,0)$ medicamentos por persona y de $3,1(1,8)$ por persona que utilizaba medicamentos. El $34,2 \%$ de la población que usaba medicamentos a diario lo hacía en número igual o superior a 4 . La media de dosis/día y del número de dosis/ número de medicamentos fue de $4,4(2,9)$ y $1,4(0,5)$ respectivamente (tabla 1$)$. 
Tabla 1

Consumo diario de medicamentos

\begin{tabular}{|c|c|c|c|c|}
\hline & Hombres & Mujeres & $p$ & Total \\
\hline ¿Toma usted algún medicamento a diario? & $n=135$ & $n=227$ & & $N=362$ \\
\hline \% sí & 72,6 & 89,4 & 0,001 & 83,1 \\
\hline ¿Cuántos? media (DE) & $2,2(2,1)$ & $2.8(1.9)$ & 0,02 & $2,6(2,0)$ \\
\hline ¿EN CASO AFIRMATIVO, cuántos toma? $\%$ & $n=98$ & $n=203$ & & $N=301$ \\
\hline Uno & 20,4 & 19.7 & 0,54 & 19,9 \\
\hline Dos & 26,5 & 28,1 & & 27,6 \\
\hline Tres & 22,5 & 16,2 & & 18,3 \\
\hline Cuatro o más & 30,6 & 36,0 & & 34,2 \\
\hline Media (DE) & $3,1(1,9)$ & $3,1(1,8)$ & 0,93 & $3,1(1,8)$ \\
\hline \multicolumn{5}{|l|}{ Cálculo del número $\left(\mathrm{n}^{\circ}\right)$ diario de dosis } \\
\hline Media (DE) 4,6 (3.2) & $4,4(2,8)$ & 0,58 & & $4,4(2,9)$ \\
\hline \multicolumn{5}{|l|}{ Relación n. ${ }^{\circ}$ dosis/ n. ${ }^{\circ}$ medicamentos } \\
\hline Media (DE) & $1,5(0,6)$ & $1,4(0,5)$ & 0,30 & $(0,5)$ \\
\hline Mediana & 1,5 & 1,3 & 1,4 & 1,3 \\
\hline
\end{tabular}

(DE): desviación estándar.

Los medicamentos más usados fueron los antihipertensivos $(41,8 \%)$, los vasodilatadores periféricos dirigidos a la circulación cerebral, también llamados «vasodilatadores cerebrales» $(21,3 \%)$, las benzodiacepinas y nuevos hipnóticos $(17,4 \%)$ y los antiinflamatorios no esteroideos (AINES) $(14,1 \%$ ) (tabla 2).

El 91,3\% de los sujetos con consumo diario de medicamentos utilizaba uno o más desde hacía al menos un año, y el 46,1\% desde hacía al menos cinco años (tabla 3).

El 20,7 \% de los sujetos entrevistados utilizaba medicación a demanda y el 7,3\% de la población consumía medicación de forma periódica pero no diaria (tabla 4). Los medicamentos a demanda más frecuentes fueron los analgésicos (36 de 74 sujetos), particularmente los AINES (11 de 74), las benzodiacepinas (10 de 74 ) y los alcalinos e inhibidores del receptor $\mathrm{H}_{2}$ de la histamina ( 8 de 74). Los medicamentos no diarios más frecuentes fueron las preparaciones de vitamina D ( 5 de 26 sujetos).
Tanto la prevalencia del consumo como el promedio de medicamentos fue mayor en las mujeres $(89,4 \%$ y 2,8$)$ que en los hombres $(72,6 \%$ y 2,2$)$, de forma estadísticamente significativa (tabla 1). Sin embargo, en los sujetos consumidores no existieron diferencias significativas por género en el promedio (3,1 medicamentos por persona mayor). Sólo los antiagregantes no aspirina, los medicamentos antiulcerosos, los respiratorios, y los comunmente dirigidos a la hipertrofia benigna de próstata, fueron consumidos por los hombres en mayor proporción que las mujeres. En cambio las mujeres utilizaban vasodilatadores periféricos dirigidos a la circulación cerebral $(26,0 \%)$ y AINES $(17,6 \%)$, en mayor proporción que los hombres.

No existieron diferencias relevantes por género en el consumo de medicamentos de forma crónica (tabla 3 ). Las mujeres utilizaban medicamentos a demanda en mayor proporción $(23,1 \%)$ que los hombres $(16,5 \%)$, $\mathrm{p}=0,15$ (tabla 4$)$. 
Tabla 2

Consumo diario de medicamentos por grupos terapéuticos

\begin{tabular}{|c|c|c|c|}
\hline$\%$ & $\begin{array}{c}\text { Hombres } \\
n=135\end{array}$ & $\begin{array}{c}\text { Mujeres } \\
n=227\end{array}$ & $\begin{array}{c}\text { Total } \\
N=362\end{array}$ \\
\hline \multicolumn{4}{|l|}{ Sistema nervioso central } \\
\hline Antidepresivos * & 1.5 & 9,7 & 6,6 \\
\hline Benzodiacepinas y nuevos hipnóticos ** & 8,1 & 22,9 & 17,4 \\
\hline Neurolépticos & 3,7 & 5,7 & 5,0 \\
\hline Vasodilatadores cerebrales $* *$ & 13,3 & 26,0 & 21,3 \\
\hline Antivertiginosos & 0.0 & 2,2 & 1,4 \\
\hline Anticonvulsivantes & 3.0 & 0,5 & 1,4 \\
\hline Antiparkinsonianos & 0,0 & 0,9 & 0,5 \\
\hline \multicolumn{4}{|l|}{ Aparato cardiocirculatorio } \\
\hline Antihipertensivos & 37,6 & 44,2 & 41,8 \\
\hline Diuréticos & 10,2 & 13,7 & 12,5 \\
\hline Digoxina & 4,4 & 5,3 & 5,0 \\
\hline Otros antiarrítmicos & 0,0 & 1,8 & 1,1 \\
\hline Antiagregantes no aspirina $* *$ & 6,6 & 1,3 & 3,3 \\
\hline Aspirina & 9,4 & 12,3 & 11.3 \\
\hline Venodilatadores & 4.3 & 9.2 & 7,5 \\
\hline Vasodilatadores arteriales periféricos & 5,8 & 6,6 & 6.3 \\
\hline Betabloqueantes & 1,5 & 1,8 & 1,7 \\
\hline \multicolumn{4}{|l|}{ Aparato digestivo } \\
\hline Laxantes & 2,2 & 2,6 & 2,5 \\
\hline Alcalinos & 6,7 & 4,9 & 5.6 \\
\hline Anti histamínicos $\mathrm{H}_{2} * *$ & 11,1 & 3,5 & 6,4 \\
\hline \multicolumn{4}{|l|}{ Analgésicos } \\
\hline Antiinflamatorios no esteroideos $* *$ & 8.1 & 17,6 & 14,1 \\
\hline Paracetamol & 2,9 & 5,7 & 4.7 \\
\hline Dipirona magnésica/hyoscina & 0,7 & 2.2 & 1,7 \\
\hline Compuestos con codeína & 0.7 & 2,2 & 1,7 \\
\hline Tramadol & 0,0 & 2,7 & 1,7 \\
\hline \multicolumn{4}{|l|}{ Aparato respiratorio } \\
\hline Broncodilatadores ** & 7,2 & 1,8 & 3,9 \\
\hline Anticatarrales & 5,1 & 3,5 & 4,1 \\
\hline Oxígeno & 0.7 & 0,0 & 0,3 \\
\hline \multicolumn{4}{|l|}{ Sistema endocrino metabolico } \\
\hline Hipolipemiantes & 8,1 & 9,7 & 9,1 \\
\hline Antidiabéticos orales & 9,4 & 13.7 & 12,1 \\
\hline Ilipouricemiantes & 0.7 & 0,9 & 0,8 \\
\hline Insulina & 1,5 & 2,6 & 2,2 \\
\hline Hormonas tiroideas & 0,7 & 0,0 & 0.3 \\
\hline Progestágenos & 0,7 & 0,0 & 0,3 \\
\hline Gonadotropas & 0,7 & 0,0 & 0.3 \\
\hline Corticoesteróides orales & 1.5 & 1,3 & 1,4 \\
\hline crema o pomada & 0.7 & 0,4 & 0.6 \\
\hline Inhalados & 2,2 & 0,9 & 1,4 \\
\hline
\end{tabular}

$* p \leq 0.05$ :

$* *: p \leq 0,01$ 
Tabla 2

Consumo diario de medicamentos por grupos terapéuticos

(Continuación)

\begin{tabular}{|c|c|c|c|}
\hline$\%$ & $\underset{n=135}{\text { Hombres }}$ & $\begin{array}{c}\text { Mujeres } \\
n=227\end{array}$ & $\begin{array}{c}\text { Total } \\
N=362\end{array}$ \\
\hline \multicolumn{4}{|l|}{ Nutrientes } \\
\hline Calcio & 1.5 & 6,2 & 4,5 \\
\hline Compuestos protéicos & 0,0 & 0,9 & 0,5 \\
\hline Hierro & 2,2 & 0,9 & 1,4 \\
\hline Tiamina & 0,0 & 0,9 & 0,5 \\
\hline Piridoxina & 0,7 & 0,0 & 0,3 \\
\hline Cianocobalamina & 0,0 & 0,4 & 0,3 \\
\hline Potasio & 0,9 & 0,7 & 0,8 \\
\hline \multicolumn{4}{|l|}{ Otros } \\
\hline Antibióticos & 5,1 & 2,7 & 3,6 \\
\hline Antialérgicos/antihistamínicos $\mathrm{HI}$ & 4,4 & 1,3 & 2,5 \\
\hline Calcitonina & 0,0 & 3,5 & 2,2 \\
\hline Colirios & 4,3 & 1,8 & 2,8 \\
\hline $\begin{array}{l}\text { Medicamentos comunmente dirigidos a } \\
\text { la hipertrofia benigna de próstata ** }\end{array}$ & 5,8 & 0,0 & 2,5 \\
\hline
\end{tabular}

$*: p \leq 0,05 ; * * ; \leq 0,01$.

Tabla 3

Consumo crónico de medicamentos diarios

\begin{tabular}{|c|c|c|c|c|}
\hline & $\begin{array}{c}\text { Hombres } \\
n=98\end{array}$ & $\begin{array}{c}\text { Mujeres } \\
n=203\end{array}$ & $p$ & $\begin{array}{c}\text { Total } \\
N=301\end{array}$ \\
\hline \multicolumn{5}{|c|}{ Número de medicamentos que consume desde hace 10 más años \% } \\
\hline cero & 9,8 & 8,1 & 0,70 & 8,7 \\
\hline uno & 28,3 & 31,6 & & 30,6 \\
\hline dos & 29,4 & 25.5 & & 26.7 \\
\hline tres & 17,4 & 17,9 & & 17,7 \\
\hline cuatro o más & 15,1 & 16,9 & & 16.3 \\
\hline \multicolumn{5}{|c|}{ Número de medicamentos que consume desde hace 5 o más años \% } \\
\hline cero & 55,6 & 53,1 & 0,90 & 53,9 \\
\hline uno & 24,4 & 24,5 & & 24,5 \\
\hline dos & 15,6 & 14,6 & & 14,9 \\
\hline tres & 2,2 & 5,7 & & 4,6 \\
\hline cuatro o más & 2,2 & 2,1 & & 2,1 \\
\hline
\end{tabular}

La prevalencia del consumo diario aumentó con la edad en los hombres, pero no en las mujeres, dónde el menor consumo correspondió a las más ancianas y el mayor consumo al grupo de edad intermedio (tabla 5). A los varones de 85 o más años correspondió la mayor proporción de consumo $(100 \%)$ y el mayor promedio $(3,3)$ de medicamentos. 
Tabla 4

Consumo no diario de medicamentos.

\begin{tabular}{|c|c|c|c|c|}
\hline & $\begin{array}{c}\text { Hombres } \\
n=1.35\end{array}$ & $\begin{array}{c}\text { Mujeres } \\
n=227\end{array}$ & $p$ & $\begin{array}{c}\text { Total } \\
N=362\end{array}$ \\
\hline \multicolumn{5}{|c|}{ ¿Toma por su cuenta algún medicamento «cuándo lo necesita», esto es, a demanda? \% } \\
\hline no & 83,5 & 76,9 & 0,15 & 79.3 \\
\hline uno & 14,3 & 16,9 & & 15.9 \\
\hline $\operatorname{dos}$ & 1.5 & 5,8 & & 4.1 \\
\hline tres o más & 0,7 & 0,4 & & 0.3 \\
\hline \multicolumn{5}{|c|}{ ¿Toma usted algún medicamento periódica pero no diariamente?\% } \\
\hline no & 93,2 & 92,4 & 0,52 & 92.7 \\
\hline uno & 3,8 & 6,2 & & 5.3 \\
\hline dos & $2,,^{3}$ & 0,9 & & 1.4 \\
\hline tres & 0,7 & 0,4 & & 0.6 \\
\hline
\end{tabular}

Tabla 5

Consumo diario de uno o más medicamentos por grupos de edad

\begin{tabular}{|c|c|c|c|c|c|c|c|c|c|c|c|}
\hline & \multicolumn{3}{|c|}{$65-74$} & \multicolumn{3}{|c|}{$75-84$} & \multicolumn{3}{|c|}{$\geq 85$} & \multirow[t]{2}{*}{$p$} & \multirow[t]{2}{*}{$p^{*}$} \\
\hline & $n$ & $\%$ & media $(D E)$ & $n$ & $\%$ & media $(D E)$ & $n$ & $\%$ & media $(D E)$ & & \\
\hline Hombres & 81 & 65,4 & $2,0(2,0)$ & 47 & 80,8 & $2,6(2,2)$ & 7 & 100 & $3,3(1,6)$ & 0,004 & 0,11 \\
\hline Mujeres & 105 & 89.5 & $2,6(1,8)$ & 90 & 91,1 & $3,0(2,2)$ & 32 & 84,4 & $2,5(2,1)$ & 0,56 & 0.35 \\
\hline Total & 186 & 79,0 & $2,3(1,9)$ & 137 & 87,6 & $2,8(2,1)$ & 39 & 87,2 & $2,6(2,2)$ & 0,09 & 0,09 \\
\hline
\end{tabular}

p: representa la significación estadística del cambio, de las proporciones con la edad;

$\mathrm{p}^{*}$ : representa la significación estadística del cambio de la media con la edad (ANOVA).

\section{DISCUSIÓN}

En el presente trabajo observamos un alto consumo actual y crónico de medicamentos en la población anciana. Introdujimos dos métodos para evaluar la complejidad del régimen terapéutico: el número de dosis/número de medicamentos, y la proporción de personas que consumen medicamentos prescritos periódica pero no diariamente. Con esta forma de evaluación, el régimen terapéutico de nuestra población parece suficientemente sencillo para favorecer el cumplimiento.

En nuestro país, por la gratuidad del medicamento para los mayores, no podría hablarse con propiedad del medicamento no prescrito como en otros países". Aunque el medicamento sea «autoindicado» 0 «indicado» por el farmacéutico, un familiar, vecino o amigo en su origen, en sucesivas consultas, éste acaba siendo prescrito por el médico a instancias de la persona mayor, a veces «por que lo debe en la farmacia». En este trabajo no hablaremos de medicamentos prescritos o no (porque es probable que sean casi todos prescritos aunque la primera indicación no proviniese del médico), sino de la medicación habitual del paciente y de la medicación que toma a demanda cuando aparecen ciertos síntomas.

El consumo de medicamentos (población consumidora: $83,1 \%$; promedio de medicamentos por persona mayor: 2,6 ; promedio de medicamentos por persona mayor consumidora: 3,1 ), fue muy similar al de un estudio 
en Leganés (población consumidora: 80\%; promedio de medicamentos por persona mayor: 2,6$)^{10}$, y al de otro en Albacete (población consumidora: $75,6 \%$; promedio de medicamentos por persona mayor consumidora: 3,17$)^{11}$, y semejante al reflejado en algunos estudios internacionales (población consumidora: $90 \%$; promedio de medicamentos por persona mayor: $2,6-3,8)^{12,13}$. En cambio, el consumo fue elevado al comparar con otros estudios nacionales y extranjeros, tanto en la proporción de población consumidora $(58,1-73 \%)^{14-19}$ como en el promedio de medicamentos por persona mayor $(1,7-2,3)^{18,20-22}$. Sin embargo, la heterogeneidad metodológica hace difícil interpretar las diferencias existentes: distinta edad de inclusión de los sujetos (estudios sobre mayores de 60 años ${ }^{14,21}$ o de 75 años ${ }^{22}$, distinto modo de obtener la información (recetas prescritas ${ }^{19}$ o entrevistas domiciliarias ${ }^{14-18,20,21,23}$, exclusión de cicrtas medicaciones (ej.: tópicas ${ }^{22}$, distinto período de estudio; nuestro trabajo interroga por el consumo habitual, otros en las últimas dos semanas $^{14-16,18}$, en la última semana ${ }^{24} \mathrm{o}$ incluso en el día previo ${ }^{17}$ a la entrevista).

Casi todos los estudios coinciden con el nuestro en que las mujeres consumen medicamentos en mayor proporción que los hombres ${ }^{10,11,15-18,20,22,23,26-29}$. Probablemente se deba a que están más incapacitadas, perciben peor su salud, y tienen mayor prevalencia de dolor y síntomas de la esfera neurológica y afectiva que los hombres ${ }^{8}$ (pérdida de memoria, tristeza o insomnio). Sin embargo al excluir los que no utilizaban medicamentos, la media fue igual en los varones que en las mujeres ${ }^{3,1}$. Ello probablemente indica que el consumo se produce en personas enfermas y sintomáticas en igual medida, independientemente del género, y que la morbilidad prevalece más en las mujeres.

Se ha estimado que el $40-45 \%$ de los ancianos no consumen los medicamentos como se les ha indicado ${ }^{30,31}$. Ello puede ser aún más frecuente con los medicamentos que han de tomarse 364 veces al día y en aquellos que no se consumen a diario: puede ser más fácil recordar tomar un sólo comprimido diario de un medicamento que tomar un comprimido los días pares de la primera quincena de cada mes. La variable número de dosis/número de medicamentos pretende indicar la complejidad del régimen terapéutico. En nuestro estudio los sujetos guardaron una relación dosis/medicamentos de 1,4 (esto es, la mayor parte de los medicamentos diarios se tomaban en una o dos dosis), y sólo el 7,3\% de la población utilizaba medicamentos prescritos periódica pero no diariamente. Por tanto, el régimen de medicamentos parece adecuado para un óptimo cumplimiento'.

La distribución por grupos terapéuticos fue semejante a otros estudios donde el mayor consumo se observa en agentes cardiovasculares, dirigidos al sistema nervioso central y analgésicos/antiinflamatorios ${ }^{10,11,17 \text { - }}$ 19,21,22,29,32,33, reflejo de las enfermedades más prevalentes en los ancianos. Es posible observar algunas diferencias rclacionadas con el momento del estudio; así, en nuestro trabajo el consumo de diuréticos $(12,5 \%)$ fue menor que en otros realizados en la década de los 80 $(36 \%)^{22}$, antes de la aparición de los nuevos antihipertensivos. El alto consumo de «vasodilatadores cerebrales», de dudosa eficacia terapéutica, puede explicarse por la alta prevalencia de queja subjetiva de pérdida de memoria, sobre todo en las mujeres de nuestra población ${ }^{8}$. En otra muestra poblacional española, el consumo de estos fármacos ascendió a la tercera parte de la población estudiada ${ }^{23}$. Una tercera parte de los ancianos que consumen medicamentos estaban polimedicados (toman 4 ó más medicamentos), hecho que también se ha observado en otros estudios ${ }^{24,34}$. Reducir la prevalencia de consumo de estos y otros medicamentos con escaso valor terapéutico posiblemente resultaría en menor polifarmacia.

Algunas diferencias por género en cuanto al tipo de medicamentos (el mayor consumo de agentes dirigidos al sistema nervioso central y analgésicos por las mujeres, y de más 
fármacos para problemas respiratorios en los hombres) se observan también en otros ancianos españoles ${ }^{10,18,29}$, lo que refleja la distribución por género de los problemas de salud a los que van dirigidos.

Entre los muchos factores que pueden influir en el consumo de medicamentos de poblaciones limitadas figuran los hábitos de prescripción de los facultativos que trabajan en ellas. En nuestro caso, dos médicos de familia atienden a la población. No cabe pensar que, dado el elevado consumo, tiendan a polimedicar a sus pacientes más que otros facultativos. Probablemente el método empleado instando a la persona para que no olvidase mostrar ningún medicamento de los que utilizaba puede explicar el elevado consumo encontrado, que además es parecido al de otros estudios con metodología similar ${ }^{10,11}$.

En los hombres, la proporción que utilizaba medicamentos diarios y el promedio de los mismos aumentó con la edad, pero no en las mujeres, dónde la mayor proporción de consumidoras (y el mayor promedio) se encontró en el grupo intermedio de edad. Este mismo patrón se observa en los ancianos de Leganés ${ }^{10}$. En algunos estudios, las diferencias de consumo de fármacos entre hombres y mujeres se acortan en aquellos mayores de 85 años ${ }^{10,19,35}$; en nuestro estudio, en realidad se invierten: las mujeres de 85 años utilizaban fármacos en menor proporción y promedio $(84,4 \%$ y 2,5 respectivamente) que los varones de su misma edad ( $100 \%$ y 3,3 respectivamente).

Es difícil discernir si la proporción de medicamentos que se consumen sin pauta fija (sólo cuando aparecen ciertos síntomas) $(20 \%)$ es o no elevada. En otro estudio ${ }^{36}$, sólo el $10 \%$ de la población utilizaba medicamentos según necesidad, pero se trata de una muestra combinada de sujetos ingresados en residencias de ancianos y de individuos que acuden a centros de salud, por lo que seguramente se trata de personas más enfermas y más dependientes del consumo regular de medicamentos. Aunque los medicamentos deben ser prescritos por el médico, consumir ciertos grupos terapéuticos según necesidad y con su conocimiento, no es sólo una práctica fucra de toda cucstión, sino rccomendable para el autocuidado de ciertas enfermedades crónicas como la enfermedad degenerativa articular o la obstrucción crónica al flujo aéreo ${ }^{37}$. La alta proporción de sujetos que tomaban medicación crónica se justifica por la alta prevalencia de enfermedades crónicas en los ancianos, y se observa también en otros estudios ${ }^{38}$.

En conclusión el consumo actual y crónico de medicamentos es de considerable magnitud. No creemos, sin embargo, que sea mayor que en otras poblaciones españolas, pues en estudios con similar metodología el consumo es muy parecido. En relación con los escasos estudios extranjeros con base poblacional de que disponemos, cualquier comparación sería aventurada. Los agentes que más se consumen son los dirigidos al sistema cardiovascular, al sistema nervioso central y los analgésicos/ antiinflamatorios. El régimen terapéutico se estima adecuado para facilitar el cumplimiento por parte de la persona mayor, aunque el elevado consumo y la polifarmacia pueden dificultarlo. Deben constituirse y continuarse medidas dirigidas a favorecer un mejor uso de medicamentos en los ancianos (correcta indicación del medicamento, buen cumplimiento, evitar en lo posible la polifarmacia), que aseguren que el uso de fármacos en el anciano mejore, y no empeore como ocurre en ocasiones, los problemas de salud de esta población.

\section{AGRADECIMIENTOS}

A todas las personas mayores del municipio de Villanueva de los Castillejos (Huelva), por su colaboración en la realización del presente trabajo, y al departamento de Epidemiología y Bioestadística de la Escuela Nacional de Sanidad, por la ayuda en la elaboración de este manuscrito. 


\section{BIBLIOGRAFÍA}

1. The Royal College of Physicians of London and the British Geriatric Society. Medication for the elderly. London: The Royal College of Physicians of London; 1984.

2. Nolan L, O'Malley K. Precribing for the elderly. Part I. Sensitivity of the elderly to adverse drug reactions. J Am Geriatr Soc 1988; 36:142-9.

3. Williamson J, Chopin JM. Adverse reactions to prescribed drug in the elderly: a multivariate investigation. Age Ageing 1980; 9: 73-80.

4. Gurwitz JH, Avorn J. The ambiguous relation between aging and adverse drug reactions. Ann Intern Med 1991; 114: 956-66.

5. Montamat SC,Cusack B. Overcoming problems with polipharmacy and drug misuse in the elderly. Clin Geriatr Med 1992; 8: 143-158.

6. Bernstein LR, Flokman S, Lazarus RS. Characterization of the use and misuse of medications by an elderly, ambulatory population. Med Care 1989; $27: 654$.

7. Klein LE, German PS, Levine DM, Feroli ER, Ardery J. Medication problems among outpatients. Arch Intern Med 1984; 144: 1185-1188.

8. Valderrama E. Situación sociosanitaria de los ancianos de una comunidad rural. [Tesis doctoral]. Madrid. Universidad Autónoma de Madrid; 1997.

9. Stoehr GP, Ganguli M, Seaberg EC, Echement DA, Belle S. Over-the-counter medication in an older rural community: the MoVIES project. $\mathbf{J}$ Am Geriatr Soc 1997; 45: 158-165.

10. Zunzunegui MV, Béland F, Recalde JM. La utilización de medicamentos en las personas mayores que residen en su comunidad. Rev Esp Geriatr Gerontol 1997; 32: 109-115.

11. López-Torres J, Cerdá R, Fernánde $\iota$ C, Requena M, Fernández C, Otero A. Factores asociados al consumo de medicación crónica en personas ancianas. Med Clin (Barc) 1997; 108: 572-576.

12. Nolan L, O'Malley K. Prescribing for the elderly: Part II. Prescribing patterns: differences due to age. J Am Geriatr Soc 1988; 36: 245-254.

13. Chutka DS, Evans JM, Fleming CD, Mikkelson BG. Drug prescribing for elderly patients. Mayo Clin Proc 1995; 70: 685-693.

14. Ministerio de Sanidad y Consumo. Uso de medicamentos en la tercera edad. Situación en España. Monografía de Divulgación n. ${ }^{\circ}$ 4: Madrid; Ministerio de Sanidad y Consumo; 1989.
15. Ministerio de Sanidad y Consumo. Encuesta Nacional de Salud de España 1987. Madrid: Ministerio de Sanidad y Consumo; 1989.

16. Ministerio de Sanidad y Consumo. Encuesta Nacional de Salud de España 1995. Madrid: Ministerio de Sanidad y Consumo; 1996.

17. Vila A, Martín H, Gisbert C, Pardo C, Espinosa

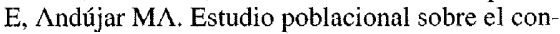
sumo de fármacos entre la población mayor de 65 años. Rev Gerontol 1993: 3: 25-28.

18. Vérez L, Fernández MC, Gude F, Tato A, Rey J, Beceiro $F$. Consumo de fármacos en ancianos y su relación con variables socioeconómicas y de salud. Rev Esp Geriatr Gerontol 1997; 32: 151-155.

19. Jörgensen TM, Isacson DGL, Thorslund M. Prescription drug use among ambulatory elderly in a swedish municipality. An Pharmacother 1993; 27: 1120-1125.

20. Eiroa P. Valoración multidimensional de la tercera edad en el municipio de Vigo. Santiago de Compostela: Universidad de Santiago de Compostela; 1994.

21. Vega S, López L, Bermejo F, de la Rosa L, Bustos $\mathrm{M}$, Sampedro MD et al. Consumo de fármacos en población mayor de 60 años en una zona tural. Aten Primaria 1996; 17: 496-500.

22. Hendriksen $\mathrm{C}$, Lund $\mathrm{E}$, Strømgård $\mathrm{E}$. Intake of drugs annong elderly people in a Danish municipality, Rødovre. Acta Med Scand 1983; 214: 6771.

23. García de Blas F. Salud general en la serie. En: Bermejo F, (ed). Nivel de salud y deterioro cognitivo en los ancianos. Fundación Caja de Madrid. Barcelona: SG editores. 1993 p.p 78-98.

24. Conselleria de Sanitat i Consum. Encuesta de Salud de la Comunidad Valenciana 1990-1991. Valencia: Conselleria de Sanitat y Consum; 1993.

25. Hale WE, Marks RG, Stewart RB. Drug use in a geriatric population. J Am Geriatr Soc 1979; 27: 374-377.

26. Koch $\mathrm{H}$. Drug utilization in office practice by age and sex of the patient: National Ambulatory Medical Care Survey, 1980. Advanced data from vital and health statistics, no. 81, 1982.

27. Landahl S. Drug treatment in 70-82-year-old persons. Acta Mcd Scand 1987; 221: 179-184.

28. Valkenburg IIA. Epidemiologic considerations of the geriatric population. J Gerontol 1988; 34 (supl 1): $2-10$. 
29. Servicio de Planificación del Departamento de Salud del Gobierno de Navarra. ¿Cómo estamos de salud? Pamplona: Gobierno de Navarra. Departamento de Salud; 1993.

30. Hurd PD, Butcovich SL. Compliance problems and the older patient: assessing functional limitations. Drug Intell Clin Pharm 1986; 20: 228-231.

31. Morrow D, Leirer V, Sheikh J. Adherence and medication instructions: review and recommendations. J Am Geriatr Soc 1988; 36: 1147-1160.

32. Rodríguez F, Martínez B, Saucedo R, Cobos F, García M, Luna J et al. Aspectos diferenciales sobre la prescripción de fármacos en ancianos residentes de la zona nordeste de Granada: estudio sobre 366 individuos. Med Clin (Barc) 1993:100: 24-27.

33. Wills P, Fastbom J, Claesson CB, Cornelius C, Thorslund M, Winblad B. Use of cardiovascular drugs in an older swedish population. J Am Geriatr Soc 1996; 44: 54-60.
34. Consejería de Salud de la Comunidad Autónoma de Madrid. Nuestros Mayores. Perfil sociosanitario de la tercera edad en la Comunidad de Madrid. Madrid: Consejería de Salud. Dirección general de planificación, formación e investigación. Serie Monografías núm 2; 1989.

35. Boethius $\mathrm{G}$. Recording of drug prescriptions in the County of Jämtland, Sweden. Pattern of drug usage in 16600 individuals during 1970-75.

36. García MD, Lucena MI, Ramírez JM, Andrade RJ, Sánchez F. Patrón de utilización de medicamentos en una población geriátrica ambulatoria. Rev Clin Esp 1992;191:412-415.

37. American Thoracic Society. Standards for the diagnosis and care of patients with chronic obstructive pulmonary disease. Am J Respir Crit Care Med 1995; 152: 77S-120S

38. Freer, CB. Study of medicine prescribing for elderly patients. Br Med J 1985;290:1113-1115. 